\title{
Evaluation of input devices for teleoperation of concentric tube continuum robots for surgical tasks
}

Carolin Fellmann, Daryoush Kashi, Jessica BurgnerKahrs

Carolin Fellmann, Daryoush Kashi, Jessica Burgner-Kahrs, "Evaluation of input devices for teleoperation of concentric tube continuum robots for surgical tasks," Proc. SPIE 9415, Medical Imaging 2015: Image-Guided Procedures, Robotic Interventions, and Modeling, 941510 (18 March 2015); doi: $10.1117 / 12.2076741$ 


\title{
Evaluation of Input Devices for Teleoperation of Concentric Tube Continuum Robots for Surgical Tasks
}

\author{
Carolin Fellmann, Daryoush Kashi, and Jessica Burgner-Kahrs \\ Emmy Noether Research Group CROSS, \\ Center of Mechatronics (MZH), Leibniz Universität Hannover, Hanover, Germany
}

\begin{abstract}
For those minimally invasive surgery where conventional surgical instruments cannot reach the surgical site due to their straight structure and rigidity, concentric tube continuum robots are a promising technology because of their small size (comparable to a needle) and maneuverability. These flexible, compliant manipulators can easily access hard to reach anatomical structures, e.g. by turning around corners. By teleoperating the robot the surgeon stays in direct control at any time. In this paper, three off-the-shelf input devices are considered for teleoperation of a concentric tube continuum robot: a 3D mouse, a gamepad, and a 3 degrees of freedom haptic input device. Three tasks which mimic relevant surgical maneuvers are performed by 12 subjects using each input device: reaching specific locations, picking and placing objects from one location to another, and approaching the surgical site through a restricted pathway. We present quantitative results (task completion time, accuracy, etc.), a statistical analysis, and empirical results (questionnaires). Overall, the performance of subjects using the 3D mouse was superior to the performance using the other input devices. The subjective ranking of the $3 \mathrm{D}$ mouse by the subjects confirms this result.
\end{abstract}

Keywords: Concentric tube continuum robot, teleoperation, human-machine interface, surgical robotics

\section{INTRODUCTION}

Teleoperation in surgical robotics is an effective method for robot control, i.e. the surgeon can directly command the motion through an input device. It provides a reliable and safe operation of a robot system and ensures the surgeon stays in full control of the surgery. Most established surgical robot systems such as the da Vinci Surgical System (Intuitive Surgical Inc., Sunnyvale, CA, USA) or the Sensei X Robotic Catheter System (Hansen Medical Inc., Mountain View, CA, USA) apply teleoperation as a control principle. In this paper, we consider continuum robots which are composed of multiple, elastic, and precurved tubes as shown in Figure 1. ${ }^{1,2}$ The tentacle-like motion of these robots results from elastic interaction of the precurved component tubes when they are arranged concentrically and individually translated and rotated axially.

Inherent flexibility and high degrees of freedom (DOF) of a concentric tube continuum robot at a size comparable to medical needles enables the robot to navigate through complex anatomical sites which are inaccessible by current surgical robots or conventional surgical instruments. Applications of concentric tube continuum robots are mainly in minimally invasive surgery ${ }^{2-4}$ and teleoperation is the method of choice for control. An appropriate input device for teleoperation of continuum robots should enable the surgeon to fully control the robot's maneuverability, its flexible structure, and to accurately command the desired motion. In addition, an input device should avoid fatigue of the surgeon's wrist and enable

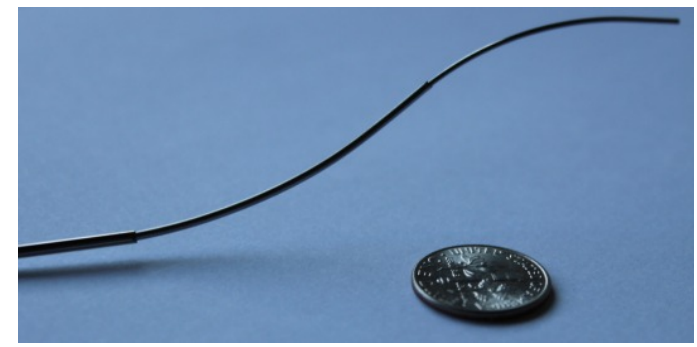

Figure 1: Concentric tube continuum robot with 3 tubes made of $\mathrm{NiTi}$ in its superelastic phase. simple handling. Up to now, input devices have not been evaluated for teleoperation of concentric tube continuum robots. The input device Geomagic Touch (formerly marketed as Phantom Omni by Sensable, now by Geomagic Inc., Rayleigh, NC, USA), which is commonly used in research as a haptic input device, has been

Corresponding author. E-mail: fellmann@mzh.uni-hannover.de. 
applied to concentric tube continuum robots with satisfactorily results. ${ }^{3}$ In the scope of this paper, we consider three alternative input devices for teleoperation: a 3D mouse, a gamepad, and a 3 DOF haptic input device. In order to evaluate the three input devices for a concentric tube continuum robot, we conducted a user study with 12 subjects. Three tasks were executed with each input device, which are based on typical surgical tasks, e.g. maneuvering along a predefined path or pick and place of tissue.

\section{METHODS}

\subsection{Kinematics of Concentric Tube Continuum Robot}

Concentric tube continuum robots consist of a minimum of two nested concentric, precurved, and elastic tubes forming the backbone of the robot. The tubes can be translated and axially rotated relative to one another at their bases, thus each tube has 2 DOF. The tubes are typically made of the shape memory alloy NiTi in its superelastic phase. The design of a robot is patient- or application-specific and depends on the parameters of each tube. ${ }^{5}$ Each tube is parameterized by its length, curvature (any arbitrary space curve), diameter, wall thickness, and material properties. In this paper, robots with $n=3$ component tubes, each with an initial straight section with length $L_{s i}$ and a subsequent curved section with length $L_{c i}$ with constant curvature $\kappa_{i}$ are considered. $i$ denotes the index of the tubes starting with the innermost tube. The total length of a tube is given by $l_{i}=L_{s i}+L_{c i}$ with $l_{1}>l_{2}>l_{3}$. For solving the forward kinematics problem a mechanics based model from Rucker et al. ${ }^{6}$ is applied. This kinematic model determines the space curve $g(s) \in S E(3)$ which describes the robot's backbone parameterized by arc length $s \in\left[0, l_{1}\right]$ for a given configuration $q=\left[\alpha_{1}, \alpha_{2}, \alpha_{3}, \beta_{1}, \beta_{2}, \beta_{3}\right]^{T}$. The homogeneous transformation $g(s)$ consists of a rotation matrix $R(s) \in S O(3)$ with its z-axis tangent to the space curve, and a translation vector $p(s) \in \mathbb{R}^{3}$ which defines the origin of the frame (see Figure 2). $\alpha_{i}$ describes the angular position of each tube's base (in radians) and $\beta_{i}$ describes the translation position e.g. the displacement of the tube relative from the entry point to the base of the tube (in meters). Rucker et. al applies Cosserat rod theory to solve the problem of the forward kinematics $g\left(\beta_{1}+l_{1}\right)=f(q)$ and considers bending and torsion of the component tubes as well as external forces and moments acting on the robot. In this paper, we consider the robot moving in free space and thus assume external forces and moment to be zero.

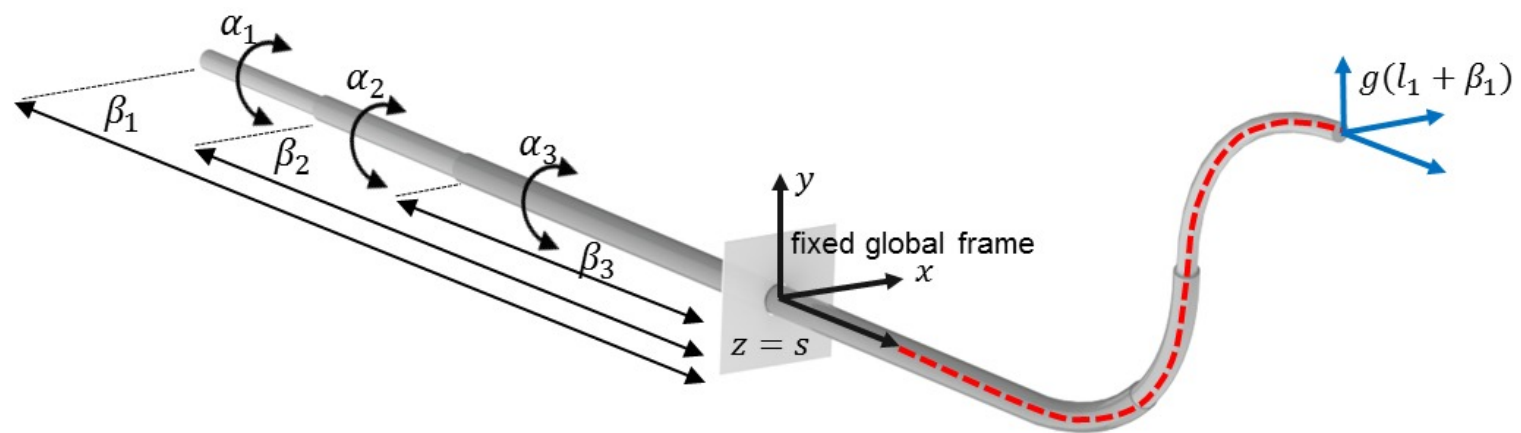

Figure 2: The configuration vector of concentric tube continuum robot with three nested tubes is $q=$ $\left[\alpha_{1}, \alpha_{2}, \alpha_{3}, \beta_{1}, \beta_{2}, \beta_{3}\right]$. Dashed line represents the arc-length parametrized space curve $g(s)$. Frame in blue defines a local coordinate system moving along the space curve in respect to the fixed global frame at the position $s=0$.

\subsection{Teleoperated Concentric Tube Continuum Robot}

A teleoperation algorithm ${ }^{3}$ is applied which uses the weighted damped least squares approach to solve the differential inverse kinematics problem. This approach determines a vector of actuator velocities $\dot{q}$ which generate the commanded motion of the robot by solving an objective function. This function is subject to competing objectives, such as accurate tracking of the input motion and damping. The robot's Jacobian required for this approach is determined using the method proposed by Rucker et al. ${ }^{7}$ Considering the objective criterion, the problem is solved by minimizing the following cost function (detailed information can be found in Deo et al..$^{8}$ ):

$$
F(\dot{q}, \dot{x})=\|\dot{x}-J \dot{q}\|_{W_{x}}^{2}+\|\dot{q}\|_{W_{q}}^{2}=\frac{1}{2}(\dot{x}-J \dot{x})^{T} W_{x}(\dot{x}-J \dot{q})+\frac{1}{2} \dot{q}^{T} W_{q} \dot{q}
$$


where $W_{x}$ is a $6 \times 6$ and $W_{q}$ is a $2 n \times 2 n$ non-negative symmetric weighting matrix associated with each of the above mentioned objectives, $\dot{x}$ is the desired $6 \times 1$ end effector velocity vector consisting of 3 angular and 3 linear components, and $\dot{q}$ is the $2 n \times 1$ vector representing the actuator velocities. Setting the derivative $\frac{\partial F}{\partial \dot{q}}$ to zero, yields to

$$
\dot{q}=\left(J^{T} W_{x} J+W_{q}\right)^{-1} J^{T} W_{x} \dot{x} .
$$

$W_{x}$ assigns priorities to end effector tracking, such that the position and orientation of the task space error have equal priorities, i.e. $1 \mathrm{~mm}$ of translational error has the same priority as $2^{\circ}$ of angular error. $W_{q}$ is associated to damp each actuator parameter independently. We define the damping matrix $W_{q}$ such that $3^{\circ}$ of rotational movement is damped as much as $1 \mathrm{~mm}$ of translational movement. The weighting matrices remain constant in all tasks and we set $W_{x}$ and $W_{q}$ as follows: $W_{x}=\operatorname{diag}\left\{10^{6}, 10^{6}, 10^{6},(90 / \pi)^{2},(90 / \pi)^{2},(90 / \pi)^{2}\right\}$ and $W_{q}=\lambda \operatorname{diag}\left\{(60 / \pi)^{2},(60 / \pi)^{2},(60 / \pi)^{2}, 10^{6}, 10^{6}, 10^{6}\right\}$, where $\lambda$ is an experimentally derived additional constant factor $\lambda=5.0$. For transforming the Cartesian velocity of the input device into the Cartesian velocity of the robot end effector $\dot{x}$ by a certain motion-scaling factor $\kappa_{s}$, the desired end effector displacement $\Delta x_{s d}$ of the next motion step is computed by taking the current master (input device) task frame $x_{m}$ and current tip frame $g_{s}\left(\beta_{1}+l_{1}\right)$ :

$$
\Delta x_{s d}=g_{s}\left(\beta_{1}+l_{1}\right)^{-1} g_{s}\left(\beta_{1}+l_{1}\right) \kappa_{s} x_{m} .
$$

When the user "clutches in" by pressing a button on the input device, the current tip frame is noticed. To determine $\dot{x}=\left[\begin{array}{llllll}\nu_{x} & \nu_{y} & \nu_{z} & \omega_{x} & \omega_{y} & \omega_{z}\end{array}\right]^{T}$, the angular velocity $\omega$ and the linear velocity $\nu$ of the desired twist motion is taken from $\Delta x_{s d}$. Given the desired $\dot{x}$, the vector of actuator velocities $\dot{q}$ is computed by applying Eq. 2 . The new actuator values $q_{i+1}$ of the next motion step results from $q_{i+1}=q_{i}+\dot{q}$. Note, that in the scope of this paper the motion-scaling factor is defined such that relationship is $1: 1$.

\subsection{Input Devices}

Three off-the-shelf and low cost input devices were selected for teleoperating concentric tube continuum robots: a 3D mouse (Space Navigator, 3DConnexion, Munich, Germany), a haptic device (Novint Falcon, Novint Technologies, Albuquerque, USA), and a gamepad (F310, Logitech, Lausanne, Switzerland). Figure 3 illustrates the defined axes for controlling the robot end effector with each input device. Each input device is connected via USB. For each input device one button is dedicated for the end effector function, i.e. opening and closing a gripper, and one button is applied to trigger the "clutch in"-mechanism. In the scope of this paper, the robot end effector's orientation is assumed constant, thus the user manipulates each input device via position control. The range of change in position ( $\mathrm{x}-, \mathrm{y}-$ and $\mathrm{z}$-direction) of each input device is mapped into the current master task frame $x_{m}$ in order to determine the desired end effector velocity. The mapping is defined for each input device independently. As long as "clutch in" is active, the range of change in position is determined until the user clutches out. Each input device has a default center position which serves as initial position in order to compute the range of change in position.

\subsubsection{D Mouse}

The 3D mouse is a 6 DOF input device which applies counterforce in opposite direction of deflection due to its elasticity. The user operates the robot with the 3D mouse's controller cap as shown in Figure 3b. The degree of the cap's deflecting in the three positioning directions determines the desired translational movement in $x_{m}$. The degree of deflection is scaled such that the translational movement of the position is in $1 \mathrm{~mm}$ increments. By tilting the cap forward and backwards, the user can move the robot end effector back and forth in z-direction. By panning the cap left and right, the x-direction of the position is defined and by panning the cap up and down the y-position of the robot end effector is moved up or down.

\subsubsection{Haptic Input Device}

The haptic input device (Novint Falcon with a standard grip) has a parallel kinematic structure connecting three arms through the gripper (forming the moving platform) used to control the device in 3 DOF. Figure $3 \mathrm{c}$ shows how the user can control the input device by manipulating the standard grip element. The moving platform can only be moved by its translational degrees of freedom, thereby its orientation remains constant. The position of the platform is computed by a combined calculation of the three encoder positions of the arms. By moving 
the grip element forward and backward determines z-direction of the platform's position. The transnational positioning in $\mathrm{x}$ - and $\mathrm{y}$-direction can be achieved by pulling the grip element to left/right (x-direction) and up/down (y-direction). The displacement of the moving platform position is mapped to $x_{m}$.

\subsubsection{Gamepad}

The gamepad is a 20 DOF input device (considering each button as 1 DOF). The user controls the robot end effector with the dual thumb stick of the gamepad as illustrated in Figure 3d. The z-axis of $x_{m}$ is respresented by pushing the right thumb stick back and forward. The left thumb stick controls the position in $\mathrm{x}$ - and $\mathrm{y}$ direction. The direction in which the left stick is pushed, determines the direction of translational movement, thereby results pushing the stick to the side to a translational movement in $\mathrm{x}$-direction and pushing the stick back and forward to a translational movement in y-direction. The degree to which the sticks are deflected is mapped to $x_{m}$.

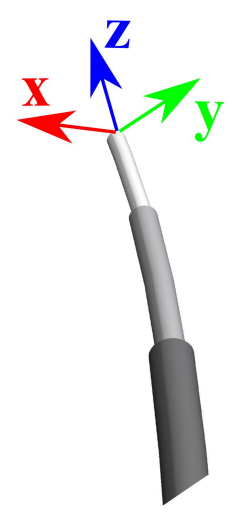

a)

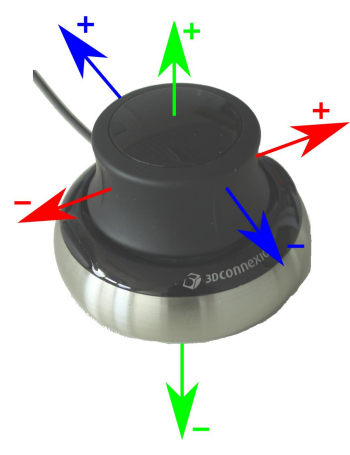

b)

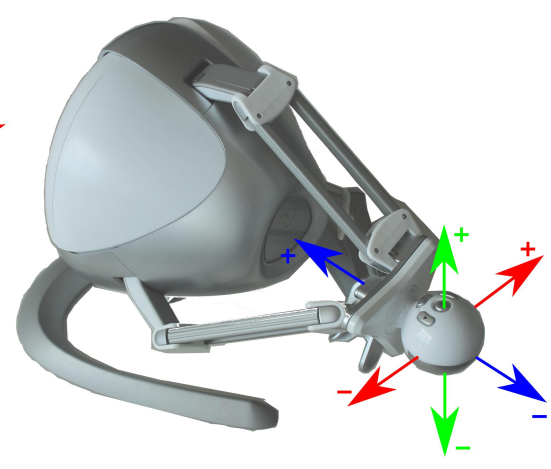

c)

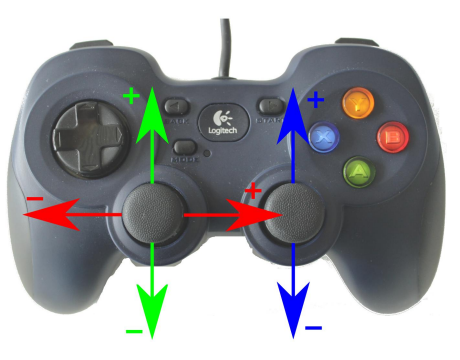

d)

Figure 3: Axis assignment for teleoperation (red: x-axis, green: y-axis, and blue: z-axis) for a) robot's end effector, b) 3D mouse, c) 3D haptic input device, and d) gamepad.

\section{EXPERIMENTAL EVALUATION}

In order to evaluate the input devices, we simulated relevant surgical tasks in minimally invasive surgery. The literature proposes transferring objects from one location to another ${ }^{9,10}$ and positioning tasks. ${ }^{10,11}$ For our experimental evaluation, we designed three tasks by adapting those existing relevant surgical scenarios.

\subsection{Tasks}

The following tasks were implemented in a simulation environment $(\mathrm{C}++$, using the Qt application framework, OpenGL and BulletPhysics libraries).

Task 1 - Position Reachability: This task relates to a surgical task which requires positioning of the robot's end effector to certain locations. The goal for the user is to teleoperate the robot's end effector to predefined locations within the workspace (presented as a red sphere as shown in Figure 4). The task is repeated 10 times with alternating locations. The user can follow the movements of the robot in two views simultaneously within the graphical user interface (GUI) of the simulation environment. The first view allows the overview of the complete scene as shown in Figure 4a. With a side view, pointing at the red sphere position, the user gets a closer look on positioning the end effector in y- and z-direction to the sphere. A color-coded grid indicates the distance of the end effector to the objects. Furthermore, a projection line supports the user to locate the robot's end effector on the sphere as shown in Figure $4 \mathrm{~b}$. 


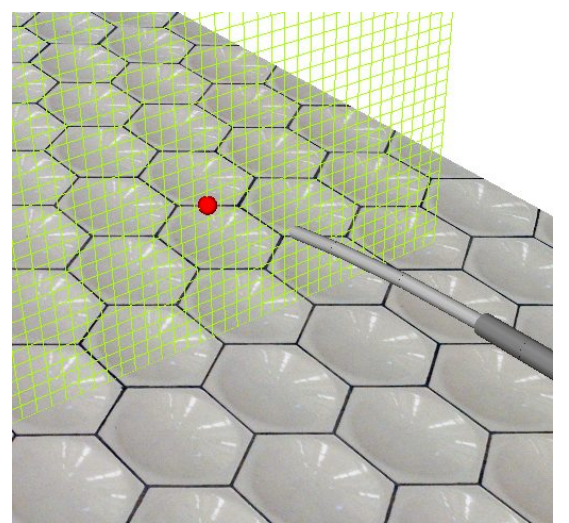

a) Main View

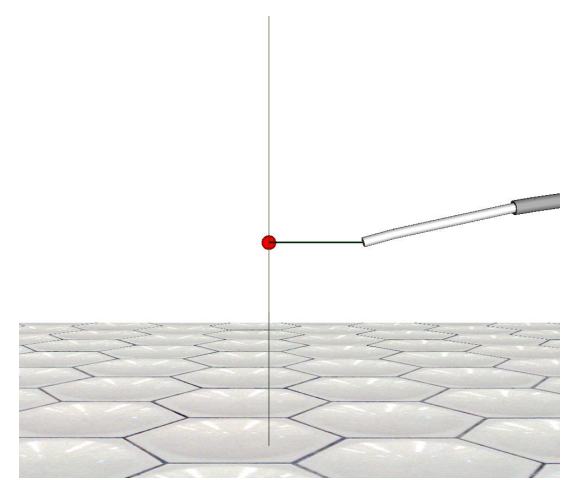

b) (Orthogonal) side view

Figure 4: Example scene for the position reachability task. a) Main view of the scene and b) side view of the scene. Green line denotes the projection line from the end effector to the red sphere.

Task 2 - Pick and Place: This task relates to a surgical task which requires transfer of tissue (e.g. tumor) from its origin to another location. The goal for the user is to pick up 4 rings individually from a stand using a gripper end effector and to transfer each ring to a bin located in front of the stand. The task is completed if no further attempts to pick up a ring are possible or if all rings are successfully transferred. Three viewpoints of the scene, including the main view, the closeup view,

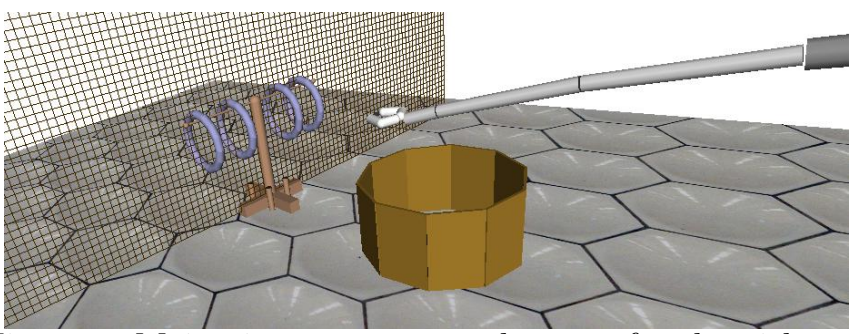

Figure 5: Main view on an example scene for the pick and place task.

and the side view, are simultaneously provided within the GUI to the user while performing the task. Figure 5 shows the main view on the complete scene. Similar to the first task, the user receives a visual support by the color-coded grid which indicates the distance of the end effector to the ring objects. The user can manually change the setting for each view, e.g. the close-up view can be focused on the gripper end effector's location relative to the rings as presented in Figure 6a and the side-view can show the positioning of the gripper end effector in front of the container (see Figure $6 \mathrm{~b}$ ). When the gripper end effector is optimally aligned to grasp a ring, the ring turns green as shown in 6 . Furthermore, a projection line is visualized between the center of the gripper end effector to the center of a ring as guide for the user.

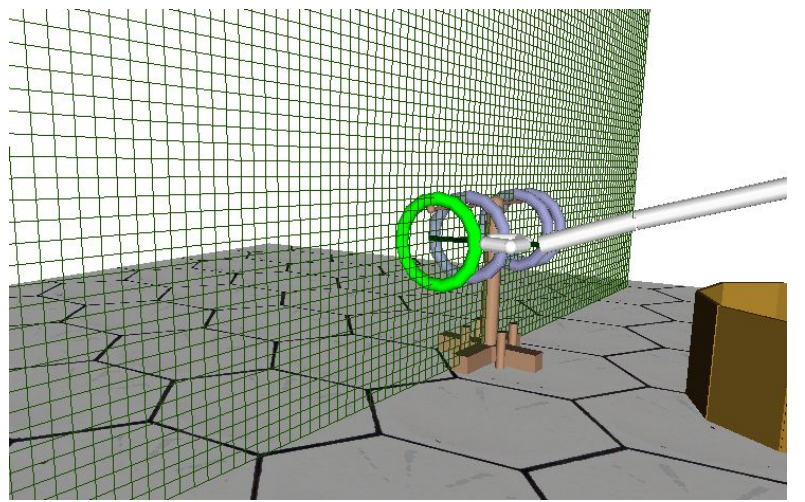

a) Closeup view

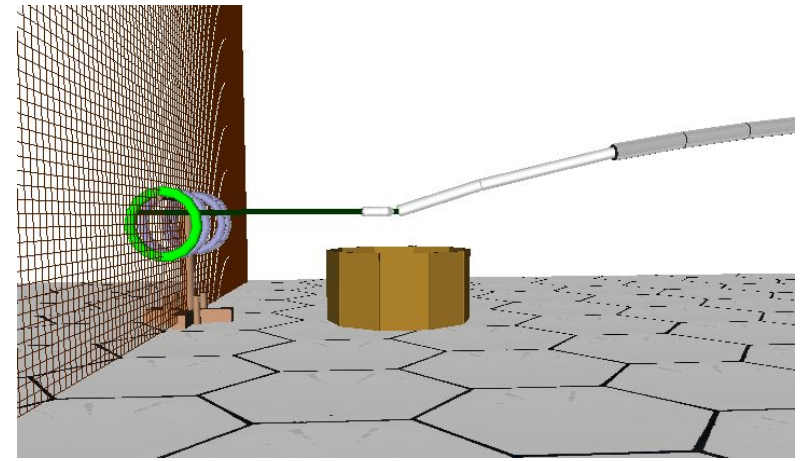

b) Side view

Figure 6: Additional viewpoints on an example scene for the pick and place task. a) Closeup view is focused on positioning the end effector in front of the rings and $b$ ) the side view shows the placing of end effector above the container. 
Task 3 - Path Following: This task relates to a surgical task where the robot has to maneuver along a tortuous path to reach the surgical site (e.g. reaching the skull base through the nose). The goal is to follow a predefined 3D path with the tip of the robot as accurately as possible. Start and finish of the task are marked by touching a red sphere at the beginning and end of the path. Figure 7 shows an example overview of the task scenario. The predefined 3D path is represented as a solid line and the robot is displayed in a red wire frame after touching the red sphere in the beginning of this task.

The GUI provides four views for the user (see Figure 8), while performing this task, as well as guiding lines for visual support. Figure 8a illustrates the "first person" view in which the camera is located at the tip of the robot. From the perspective of the robot its end effector can be moved accurately along the path by aiming the end effector circle around the white sphere representing the centerline of the 3D path. Figure 8b, c and d gives the user additional information about the scene from different camera angles.

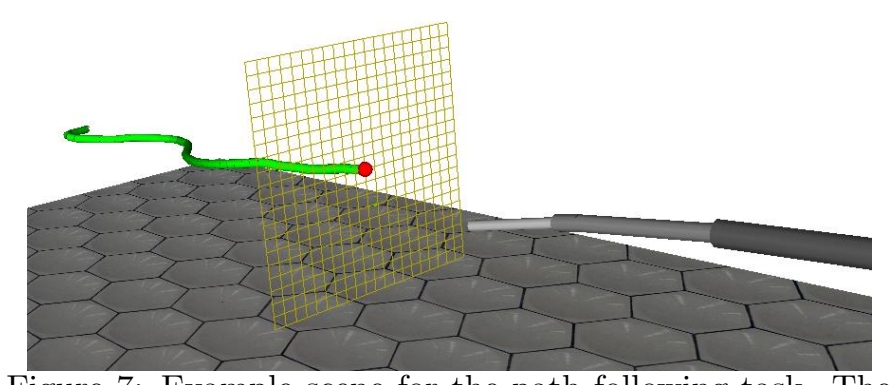

Figure 7: Example scene for the path following task. The 3D path is represented by a green solid line.

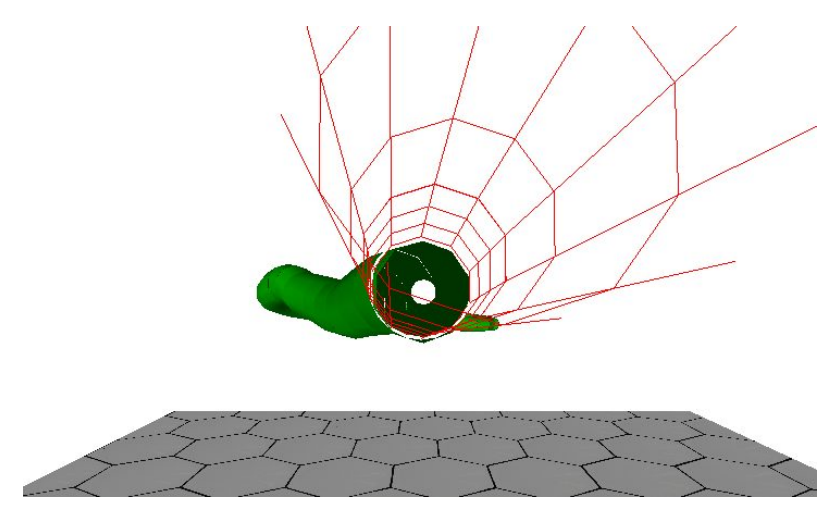

a) "First person" view

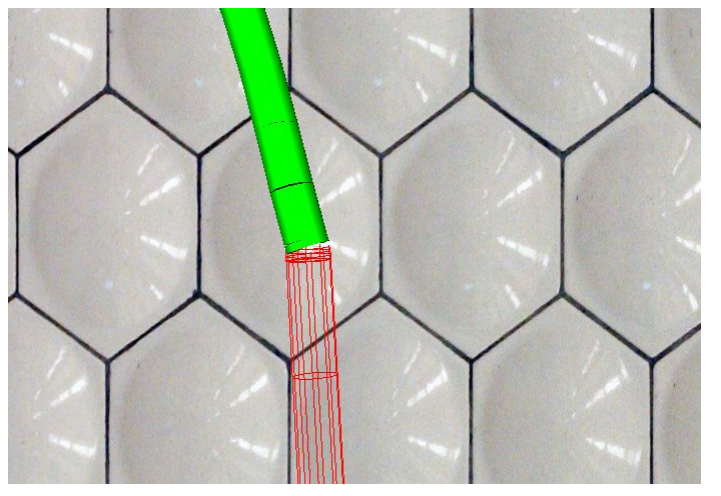

b) Top view

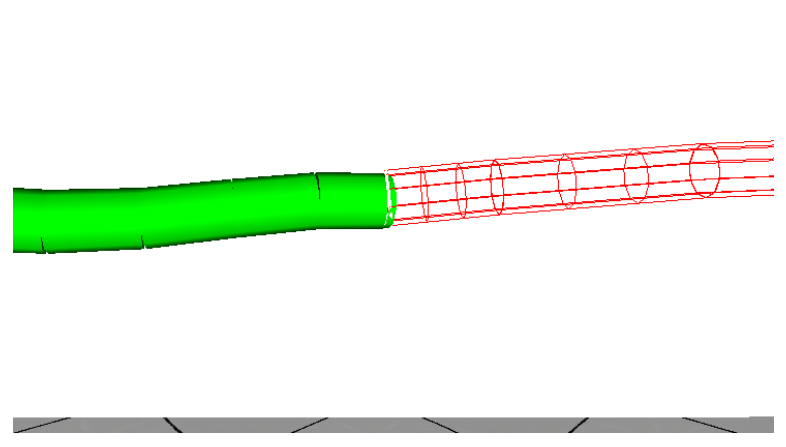

c) Side view

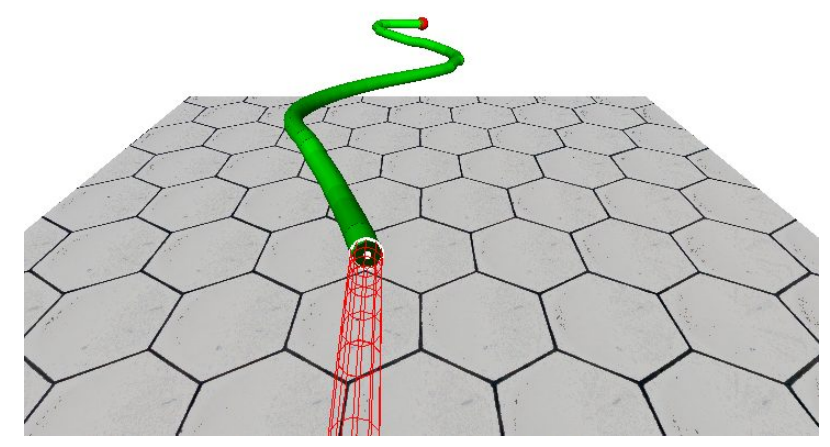

d) Main View

Figure 8: Multiple views on example scene for the path following task. The robot is displayed in a red wire frame and the predefined 3D path is represented by a green solid line. a) "First person" view shows the scene from the perspective of the robot end effector, b) top view, c) side view, and d) main view represents the current stage of the task in a variety of different orientations.

\subsection{User Study}

The user study is based on a $3 \times 3$ (input device $\mathrm{x}$ task) within-subjects design. A pre-experiment questionnaire determines the demographics of each subject, e.g. age, experience using remote controlled devices in gaming and 
using of input devices, which have more than 3 DOF. Each subject gets a brief introduction of the robot and the functionality of the input devices. The 3 tasks (see Sec. 3.1) are conducted sequentially with an initial learning phase per task. After completion of all tasks with one input device, the tasks are repeated with the other two input devices. To minimize order effects, the three input devices were counterbalanced using a Latin square i.e. the input device sequence is permuted among all subjects. After finishing all 3 tasks with all 3 input devices, each subject fills out a post-experiment questionnaire. Per subject the study takes about 45 minutes.

\subsection{Questionnaire}

The post-experiment questionnaire is divided into two parts. The first part is self-defined and contains the overall comparison of input devices for each task including a rating of a device relative to the other devices in general and selecting of the best device per task. The second part is based on the standardized questionnaire ISO 9241-400:2007 (Requirements for physical input devices). Four questions of this questionnaire are selected: mental effort, smoothness during navigation, accurate targeting and general comfort. Each question of the post-experiment subjective questionnaire is answered with a rating from 1 (best) to 5 (worst) with 3 as neutral rank.

\section{RESULTS}

A total of 12 subjects participated in the user study, including 8 male and 4 female subjects with a mean age of 27.6. Two subjects had prior experience with robot control and 6 DOF devices.

\subsection{Task Results}

The performance of each input device is measured on the task completion time (in s) as dependent variable for Task 1 and 2 and the deviation (defined by the distance of the tip to a point on the path with the same z-position in $\mathrm{mm}$ ) as dependent variable for Task 3 . Table 1 shows the results of the user study including means and standard deviation of the dependent variables over all subjects for each input

Table 1: User study results. Mean $\bar{x}$ and standard deviation $\sigma$ of measured performance over all subjects per task and input device.

\begin{tabular}{|l|l|l|l|l|l|l|}
\hline & \multicolumn{2}{|c|}{ Task 1 } & \multicolumn{2}{c|}{ Task 2 } & \multicolumn{2}{c|}{ Task 3 } \\
\hline Device & $\bar{x}[\mathrm{~s}]$ & $\sigma[\mathrm{s}]$ & $\bar{x}[\mathrm{~s}]$ & $\sigma[\mathrm{s}]$ & $\bar{x}[\mathrm{~mm}]$ & $\sigma[\mathrm{mm}]$ \\
\hline 3D Mouse & 14.0 & 7.6 & 79.3 & 28.03 & 0.14 & 0.13 \\
\hline Novint Falcon & 12.0 & 5.7 & 112.8 & 62.28 & 0.17 & 0.14 \\
\hline Gamepad & 15.4 & 8.1 & 128.4 & 68.15 & 0.20 & 0.12 \\
\hline
\end{tabular}
device. The Novint Falcon was determined as best-performed input device for Task 1 and the 3D mouse performed best for Task 2 and 3. The standard deviation of Task 2 for the 3D mouse is lower than the standard deviation of the other two input devices. This indicates that the 3D mouse performed clearly as the best input device in the second task. In addition, the non-parametric Wilcoxon-signed rank test is used to obtain statistically significant results for each task. The input devices are ranked by completion time from 1 (fastest) to 3 (slowest) for Task 1, with equally performed input devices rated similar. Here as well, the Novint Falcon has shown a significantly better performance $(z=2.84, p<0.01)$ in comparison to the 3D mouse $(z=1.13, p<0.13)$. However, analyzing the end effector paths for Task 1, we can observe that they are smoother with the 3D mouse than with the Novint Falcon. For Task 2, the input devices are also ranked by their task completion time from 1 (fastest) to 3 (slowest). The 3D mouse is determined with $z=2.39, p<0.01$ as the best-performed input device in the second task. In Task 3, the input devices are rated from 1 (best-performed) to 3 (worst-performed) by the deviation of the robots end effector from the predefined path, thus, the smallest deviation is an indicator for the best-performed input device. The 3D mouse is the best-performed input device using this metric with a significant difference $(z=2.24, p<0.025)$ in comparison to the Novint Falcon.

\subsection{Questionnaire Results}

Analysis of the rank data of the post-experiment questionnaire was conducted using a non-parametric Wilcoxonsigned rank test. The results of the following four questions were significant for determining the best input device: 
- Minimal mental effort: Novint Falcon with $z=1.82, p<0.05$

- Smoothness during navigation: 3D mouse with $z=1.94, p<0.05$

- Best in comparison with the other two: 3D mouse with $z=2.25, p<0.025$

- Ranked first for Task 1: 3D mouse with $z=2.25, p<0.025$

We point out, that the 3D mouse was ranked best for Task 1 in the subjective questionnaire, while the Novint Falcon was statistically better (see Section 4.1).

\section{CONCLUSION}

In this paper, we present the experimental evaluation of 3 off-the-shelf input devices for teleoperation of a concentric tube continuum robot. The results of our user study indicate that subjects using a 3D mouse achieved significantly better results than with other evaluated input devices. We point out that subjective preferences of the users (such as mental effort, fatigue) may influence the choice of future input devices. We suspect that the simple design and handling of the 3D mouse lead to the superior performance. Up to now, the subjects operated each input device via position control. In the future, the orientation of the input device should also be determined during the performance of relevant surgical tasks to maneuver the robot's end effector in all spatial directions. Also, the weighting matrices of the objective function should be adjusted to the respective constraints of each task in the future. Furthermore, we will extend the significance of our results by evaluating input devices in a clinical setting with surgeons and medical personnel operating a real robot. We will also consider additional input devices, such as gesture based devices and haptic devices with higher DOF. Ultimately, we foresee a dedicated input device for those special kinematic structures of continuum robots which exhibit similar properties in terms of flexibility and compliance.

\section{ACKNOWLEDGMENT}

This work was funded within the German Research Foundation's Emmy Noether Programme under award number BU 2935/1-1.

\section{REFERENCES}

[1] Gilbert, H. B., Rucker, D. C., and Webster III, R. J., "Concentric Tube Robots: The State of the Art and Future Directions," Int Symp Robot Res (2013).

[2] Dupont, P. E., Lock, J., Itkowitz, B., and Butler, E., "Design and Control of Concentric-Tube Robots," IEEE Trans Robot 26(2), 209-225 (2010).

[3] Burgner, J., Rucker, D. C., Gilbert, H. B., Swaney, P. J., Russell, P. T., Weaver, K. D., and Webster III, R. J., "A Telerobotic System for Transnasal Surgery," IEEE/ASME Trans Mech 19(3), 996-1006 (2014).

[4] Burgner, J., Swaney, P. J., Lathrop, R. A., Weaver, K. D., and Webster III, R. J., "Debulking from within: a robotic steerable cannula for intracerebral hemorrhage evacuation.," IEEE Trans Biomed Eng 60(9), 2567-75 (2013).

[5] Burgner, J., Gilbert, H. B., and Webster III, R. J., "On the Computational Design of Concentric Tube Robots: Incorporating Volume-Based Objectives," IEEE International Conference on Robotics and Automation, 1185-1190 (2013).

[6] Rucker, D. C., Jones, B. A., and Webster III, R. J., "A Geometrically Exact Model for Externally Loaded Concentric-Tube Continuum Robots," IEEE Trans Robot 26(5), 769-780 (2010).

[7] Rucker, D. C. and Webster, R. J., "Computing Jacobians and compliance matrices for externally loaded continuum robots," IEEE International Conference on Robotics and Automation, 945-950 (2011).

[8] Deo, A. S. and Walker, I. D., "Overview of damped least-squares methods for inverse kinematics of robot manipulators," Journal of Intelligent $\& 3$ Robotic Systems 14(1), 43-68 (1995).

[9] Prasad, S. M., Maniar, H. S., Soper, N. J., Damiano, R. J., and Klingensmith, M. E., "The effect of robotic assistance on learning curves for basic laparoscopic skills.," American Journal of Surgery 183(6), 702-7 (2002). 
[10] Narazaki, K., Oleynikov, D., and Stergiou, N., "Robotic surgery training and performance: identifying objective variables for quantifying the extent of proficiency.," Surgical Endoscopy 20(1), 96-103 (2006).

[11] Horise, Y., Nishikawa, A., Sekimoto, M., Kitanaka, Y., Miyoshi, N., Takiguchi, S., Doki, Y., Mori, M., and Miyazaki, F., "Development and evaluation of a master-slave robot system for single-incision laparoscopic surgery.," International Journal of Computer Assisted Radiology and Surgery 7(2), 289-96 (2012). 